\title{
UPDATE ON LYMPHOMA PATHOLOGY III LYMPHOMA CLASSIFICATION AND NODAL LYMPHOMAS
}

\author{
R W Blewitt, Consultant Pathologist \\ Royal Lancaster Infirmary
}

\section{INTRODUCTION}

Lymphoma classification has for years been confusing and at times exasperating, based solely on haematoxylin- and eosinstained histological sections. Several major advances have allowed a new and less subjective classification to be developed:

- use of immunostains

- recognition that extranodal lymphomas are special

- discovery of specific cytogenetic abnormalities in lymphomas

- better understanding of lymphocyte physiology

\section{REAL CLASSIFICATION OF LYMPHOMAS ${ }^{(1)}$}

This stands for Revised European-American Lymphoma classification. Proposed in 1994, the REAL classification is rapidly becoming accepted throughout the world. At present about 30 types of lymphoid neoplasm are categorised, but this is likely to increase in the future.

The commonest types of lymphoid neoplasm are listed in Figure 1. Leukaemias, myelomas and very rare entities have been omitted for clarity, but copies of the full classification are available from the histopathology laboratory at RLI. Several important features of the REAL classification apparent from Figure 1 will now be considered in detail: these are cell type, grade, sites and terminology.

\section{a) cell type}

Division of lymphocytes into B and T cells is a fundamental part of the REAL classification and is achieved by the use of immunostains on histological sections. Immunostains are antibodies which allow the demonstration of specific antigens in tissue sections. It turns out that the great majority of lymphomas (about $85 \%$ ) are of B-cell type. Some types of lymphoma, however, do not stain for B or T cell markers and are said to have a 'null cell phenotype': these include most types of Hodgkin's disease and some anaplastic large cell lymphomas.

\section{b) lymphoma grade}

'Grade' refers to the degree of malignancy. Low-grade lymphomas grow slowly (often described as 'indolent') and if untreated cause death after a number of years. Lowgrade lymphomas are generally composed of small lymphocytes. The tumours tend to be widespread (high stage) at presentation and most are incurable. They are also prone to undergo transformation into high-grade lymphoma. Hodgkin's disease is an exceptional type of low-grade lymphoma with a high degree of curability.

High-grade lymphomas grow rapidly and if untreated may cause death in a matter of months. On the other hand, they are potentially curable by intensive therapy. High-grade lymphomas are usually composed of large lymphocytes, and are less likely to be widely disseminated at diagnosis than low-grade lymphoma.

\section{c) sites of lymphoid neoplasia}

Lymphocytes are distributed throughout the lymphoid system (Figure 2), any part of which can be the seat of lymphoid neoplasia. Bone marrow and thymus are referred to as primary lymphoid organs, where lymphocytes are created and

\begin{tabular}{|l|l|l|l|}
\hline CELL TYPE & DIAGNOSTIC ENTITY & GRADE & SITES \\
\hline B & small lymphocytic lymphoma & low & nodes $^{*}$ \\
\hline B & mantle cell lymphoma & low & nodes, intestine \\
\hline B & foilicular lymphoma & low & extranodal \\
\hline B & marginal zone lymphoma & low & any \\
\hline T & diffuse large B-cell lymphoma & high & nodes \\
\hline T & peripheral T-cell lymphoma & high & small intestine \\
\hline T/NULL & intestinal T-cell lymphoma & high & skin \\
\hline T/NULL & anaplastic large cell lymphoma of skin & low & any \\
\hline T & anaplastic large cell lymphoma, systemic & high & skin \\
\hline B & mycosis fungoides & low & nodes \\
\hline NULL & Hodgkin's disease, lymphocyte predominance & low & nodes \\
\hline NULL & Hodgkin's disease, nodular sclerosis & low/moderate & nodes \\
\hline NULL & Hodgkin's disease, mixed cellularity & low/moderate & high \\
\hline
\end{tabular}




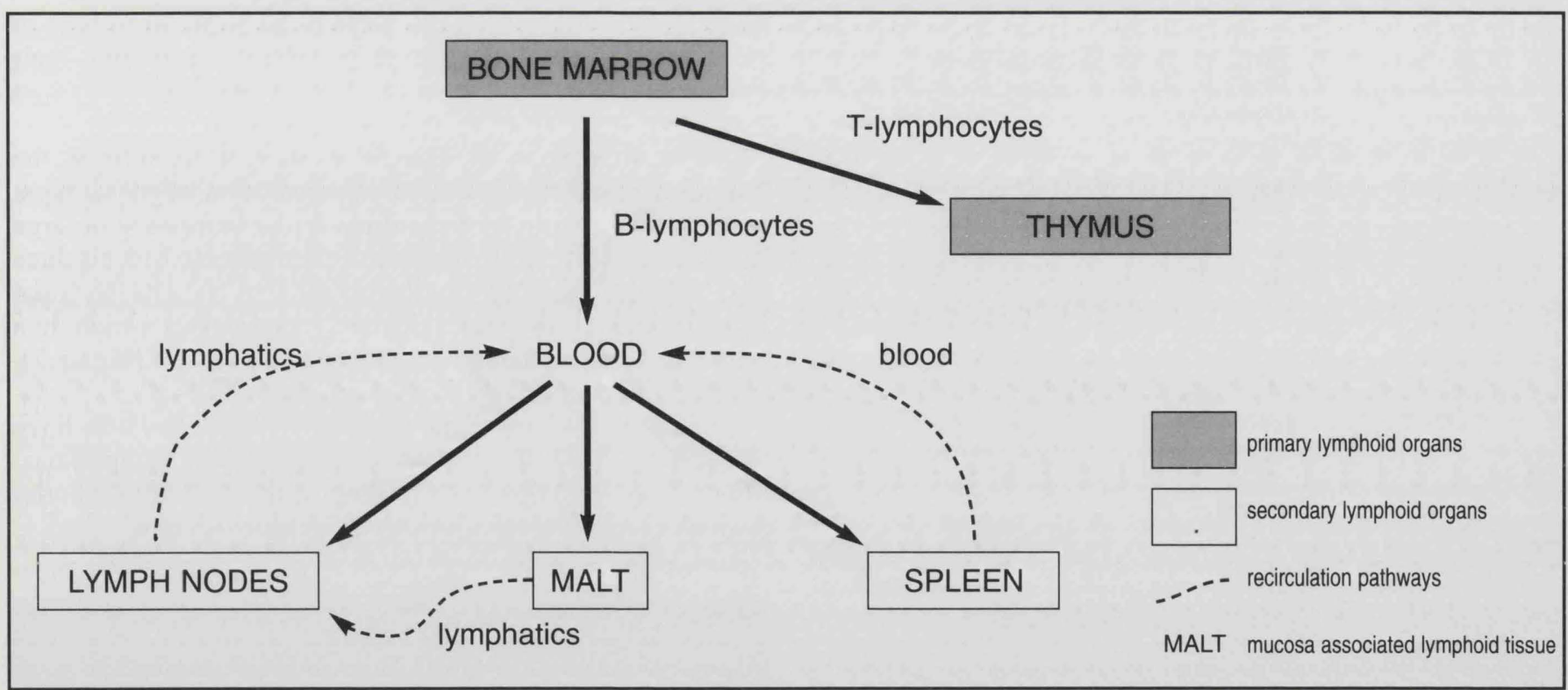

Figure 2 Diagramatic anatomy of lymphoid system and B-lymphocyte circulation

processed. Lymph nodes, spleen and MALT are secondary lymphoid organs, which represent the front line of the defensive system where antigens are recognised and immune responses mounted. MALT means mucosa-associated lymphoid tissue and is predominantly tonsils and mucosa of the intestine and bronchi ${ }^{(2)}$.

It is important to appreciate that B lymphocytes undergo continual recirculation between the secondary lymphoid organs. This is why many types of lymphoma are disseminated from the outset.

Tumours of lymphocytes in bone marrow and blood tend to produce leukaemia, whereas tumours affecting the secondary lymphoid organs usually produce lumps and appear as lymphomas.

Tumours affecting MALT sites are often referred to as extranodal lymphomas. Some lymphomas, however, are not only extranodal but also extralymphoid; lymphomas of skin, brain and stomach, for example, do not affect any normal part of the lymphoid system. Extranodal lymphomas will be considered in Part IV.

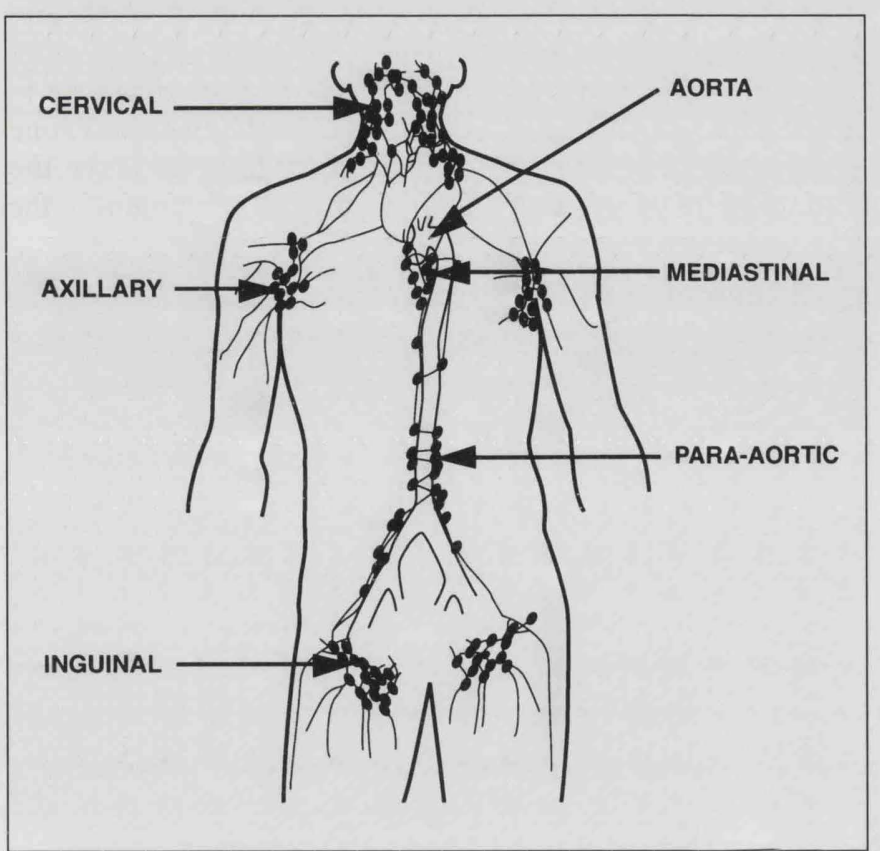

Figure 3 Diagram of lymph node groups, superficial and deep
Most lymphomas arise in lymph nodes and therefore produce palpable lumps in the neck, axillae or groins, according to the distribution of superficial lymph nodes (Figure 3). Deep lymph nodes such as para-aortic or mediastinal nodes can be affected but we require imaging techniques to identify them. Lymphomas spread from one lymph node group to the next and often involve blood, spleen and bone marrow.

\section{d) terminology of the low-grade B-cell lymphomas}

In the REAL classification (Figure 1) there are four common types of low-grade B-cell lymphoma. In order to understand the meaning of terms such as 'mantle', 'follicular' and 'marginal', we need to consider briefly how B lymphocytes develop and some aspects of lymph node structure.

\section{d(i) development of B-lymphocytes ${ }^{(2)}$}

B lymphocytes are created in the bone marrow (Figure 4). They are given antigenic specificity by a process of rearrangement of their immunoglobulin genes. This involves cutting and translocation of segments of the genes in a randomised pattern to generate all possible antigenic specifities. The resulting cell is a small lymphocyte which can produce low-affinity IgM and IgD antibody on the cell surface but is incapable of secreting high-affinity $\mathrm{IgG}$ and IgA antibody. The surface IgM acts as a receptor for its specific target antigen. The small lymphocyte then passes into the bloodstream and joins a pool of recirculating 'naïve' or 'virgin' lymphocytes which have not yet encountered the antigen to which they have specificity. The small naïve Blymphocytes are long-lived and recirculate from blood to lymph node, to lymph, and back to blood indefinitely until they encounter their target antigens (Figure 2).

It is this population of recirculating lymphocytes which is responsible for the first of the low-grade B-cell lymphoma types listed in Figure 1, small lymphocytic lymphoma. It should now be understandable that small lymphocytic lymphoma is a diffuse disease, filling up bone marrow, blood and lymph nodes, and very often leukaemic.

\section{d(ii) lymph nodes and their follicles ${ }^{(3)}$}

In the outer part of a lymph node are multiple nodular clusters of lymphocytes, the lymphoid follicles, also found in spleen and MALT lymphoid sites. Lymphoid follicles are the domain of the B-lymphocyte. In the absence of antigenic 


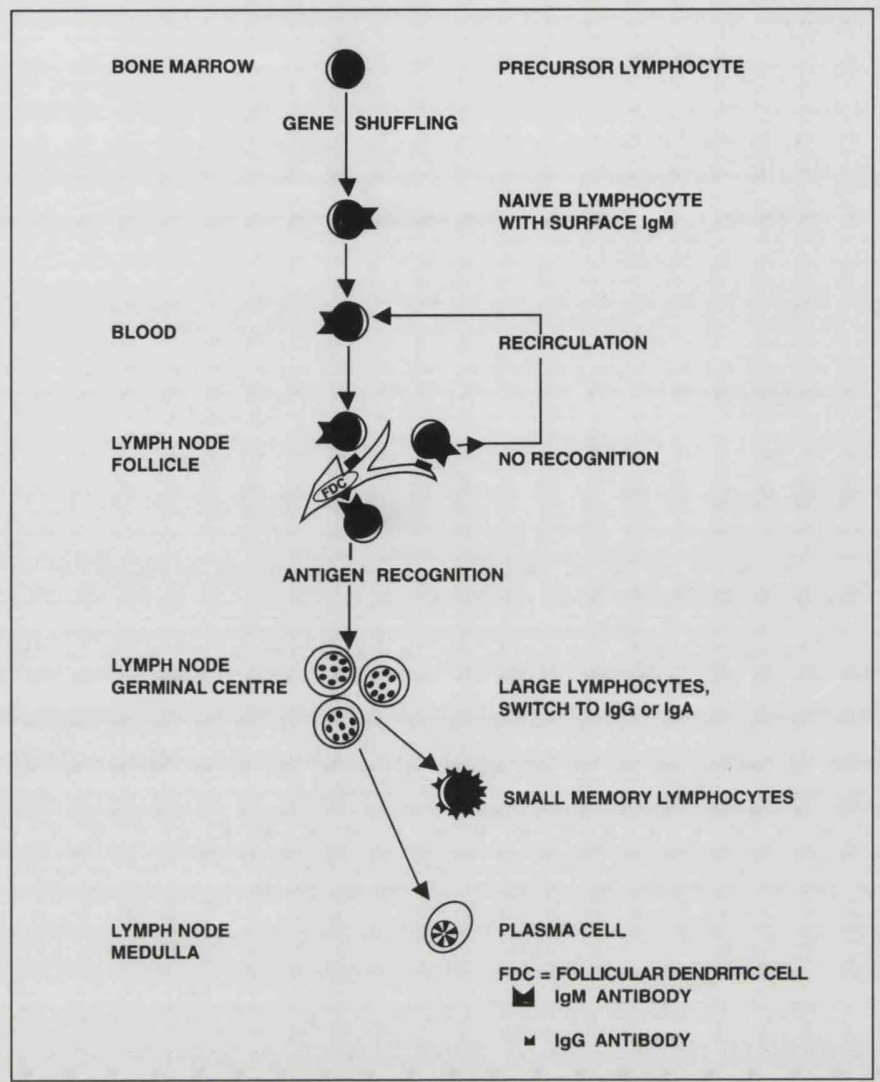

Figure 4 Maturation of B-lymphocytes

challenge, lymphoid follicles appear as dense aggregates of small lymphocytes, known as primary follicles (Figure 5). The small lymphocytes are naïve B-cells situated within a meshwork of follicular dendritic cells. Invisible in routine stains, follicular dendritic cells are demonstrable by the CD21 immunostain (Figure 6).

Follicular dendritic cells are specialised histiocytes on the surface of which captured antigen is displayed. The small

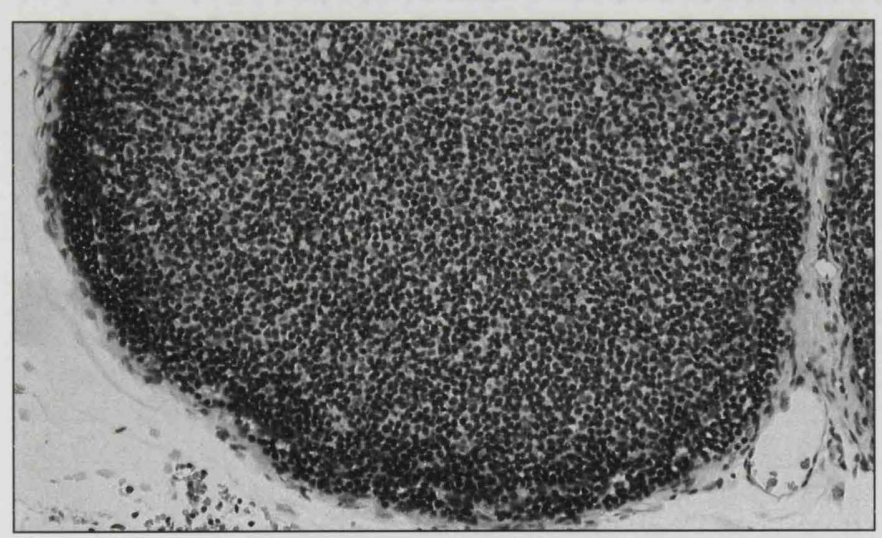

Figure 5 Primary follicle containing small lymphocytes

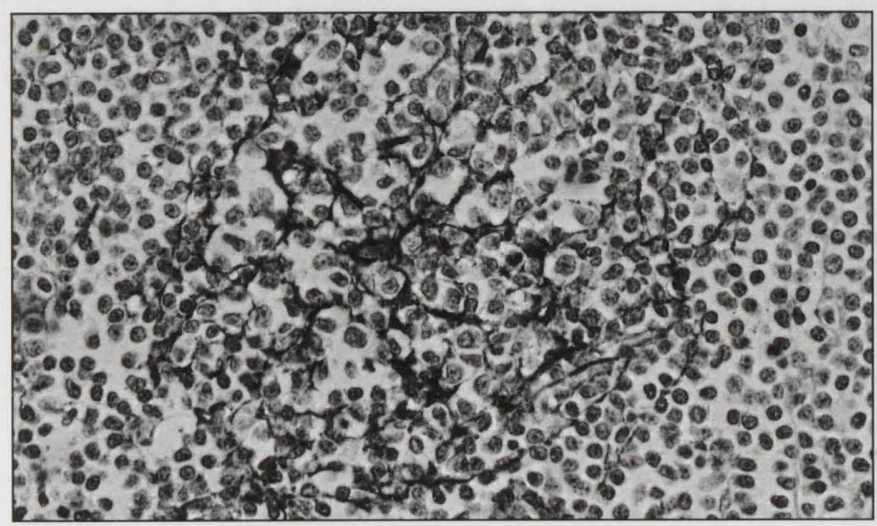

Figure 6 Follicular dendritic cells (CD21 strain) lymphocytes pause for a few days in a lymphoid follicle to 'scrutinise' the antigen display before continuing their circulation.

If a small lymphocyte recognises its specific antigen, the lymphocyte undergoes drastic changes. It undergoes a burst of proliferation to form a germinal centre composed of large lymphocytes, some of which are then selected to produce high affinity $\operatorname{IgG}$ or IgA antibody (see Part II). The germinal centre displaces the original small lymphocytes which then form the follicular mantle of the secondary follicle (Figure 7).

From the structure of the secondary follicle we now have the meanings of the next two listed B-cell lymphomas. Mantle cell lymphoma is a tumour of the follicular mantle; follicular lymphoma arises from the germinal centre.

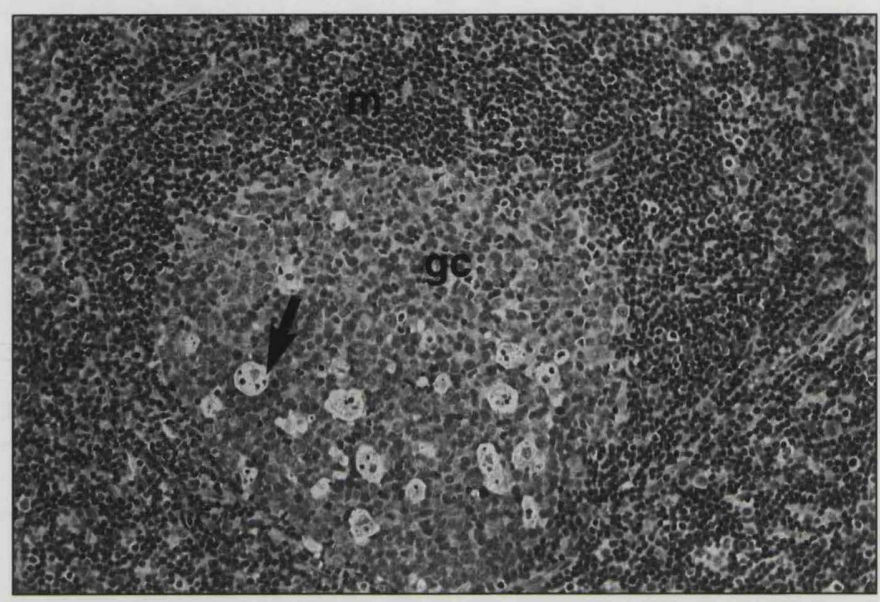

Figure 7 Secondary follicle

arrow: tingible body histiocyte.

gc: germinal centre containing large lymphocytes

$m$ : follicular mantle of small lymphocytes

d(iii) splenic lymphoid follicles and the marginal zone Lymphoid follicles are also present in the spleen, but contain an additional zone of B-lymphocytes surrounding the secondary follicle and known as the marginal zone (Figure 8 ), because it lies at the margins of the splenic B-cell areas.

Marginal zone lymphocytes are not naïve B-lymphocytes but have been through the rigours of the germinal centre. Hence the marginal zone lymphocytes are able to synthesise high-affinity IgG or IgA antibody. They have slightly more cytoplasm than other small lymphocytes and are on course to develop into plasma cells. In lymph nodes the marginal zone is very poorly developed as mature lymphocytes leave the germinal centre directly to complete their development in the

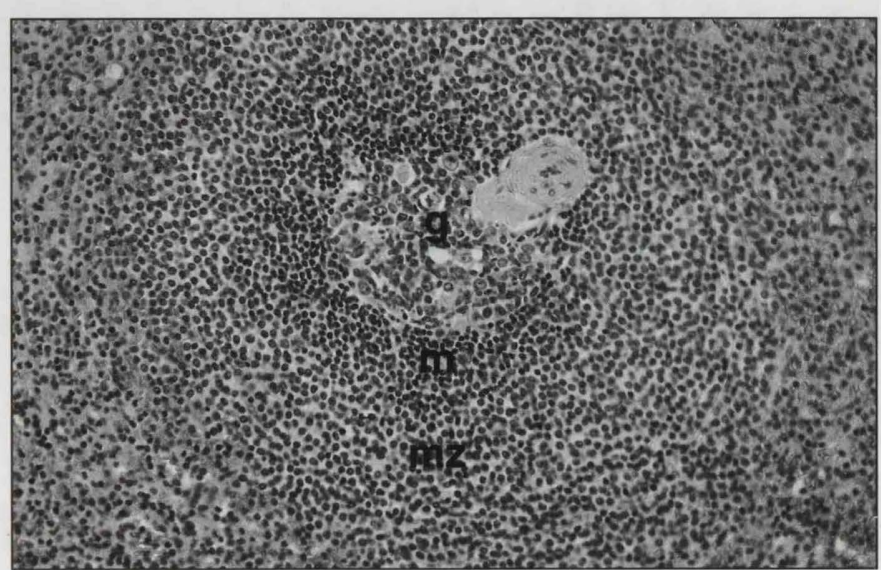

Figure 8 Secondary lymphoid follicle in spleen g: germinal centre. m: follicular mantle. mz: marginal zone 
medulla of the lymph node ${ }^{(2)}$. Marginal node lymphomas are almost always extranodal and will be considered in detail in Part IV.

We are now ready to review the ten types of lymphoma that commonly arise in lymph nodes.

\section{NODAL LYMPHOMAS ${ }^{(1)}$}

\section{Follicular lymphoma}

This is the commonest of the low-grade B-cell lymphomas. It arises almost exclusively within lymph nodes and presents with lymphadenopathy. Nevertheless, at the time of diagnosis it is usually already widespread within different lymph node groups and frequently affects bone marrow and spleen as well. The disease hardly ever occurs outside these sites.

Follicular lymphoma is a disease of adults, typically over 40 years old. The tumours grow slowly and, in common with most low-grade B-cell lymphomas, are at present incurable. An appreciable proportion undergo transformation into highgrade lymphoma, usually diffuse large B-cell lymphoma. Median survival is seven years.

Almost all cases show the $14 ; 18$ translocation leading to activation of BCL-2 (see Part II). The histology is characterised by a mass of atypical germinal centres which replace the lymph node and lack normal signs of apoptosis ('tingible body macrophages'). Diagnosis is usually straightforward. The main differential diagnosis is reactive follicular hyperplasia, but staining for BCL-2 is positive in neoplastic follicles, unlike reactive ones (Figures 9 and 10).

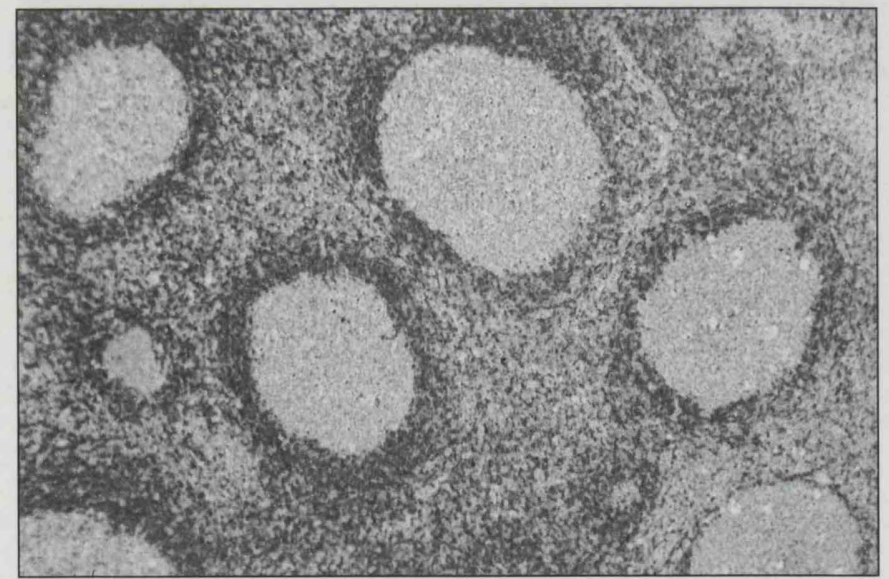

Figure 9 Reactive lymphoid follicles.

Germinal centres are unstained by $B C L-2$

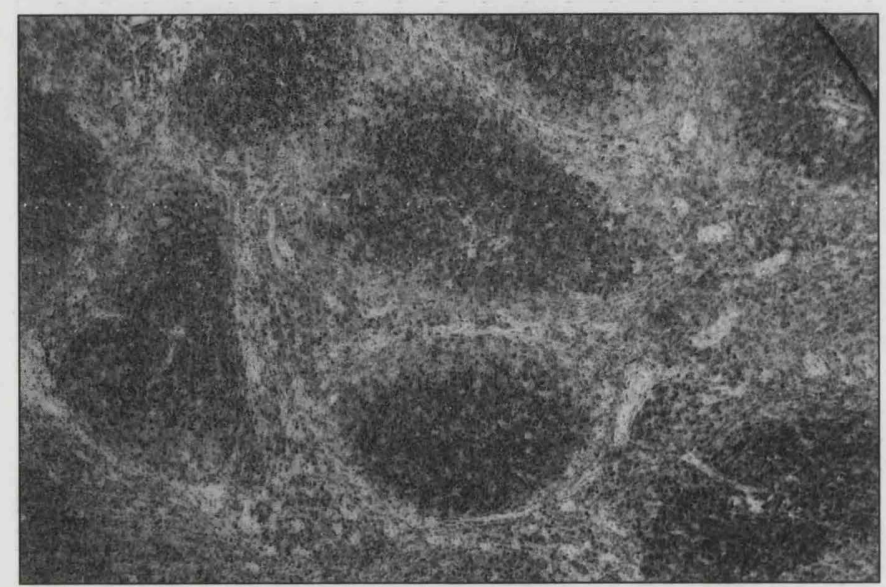

Figure 10 Follicular lymphoma.

The follicles stain for BCL-2 due to the $14 ; 18$ chromosome translocation
Mantle cell lymphoma

Mantle cell lymphoma should be thought of as the low-grade B-cell lymphoma with a bad prognosis. The least common of the low-grade B-cell lymphomas, mantle cell lymphoma has been recognised only relatively recently and is still probably underdiagnosed.

This tumour affects an older age group than follicular lymphoma and also presents with disseminated disease. Unlike follicular lymphoma, mantle cell lymphoma is often extranodal, frequently affecting the gastrointestinal tract and the tonsillar area (Waldeyer's ring) (see Part IV).

Mantle cell lymphoma is more aggressive than the other low-grade B-cell lymphomas and has the worst prognosis of this group. On the other hand, the tumour does not seem to undergo high-grade transformation. Median survival is four years.

Most cases will show the $11 ; 14$ chromosome translocation leading to overproduction of cyclin D1 which drives the mitotic cycle. The histology is of a monotonous infiltrate of small lymphocytes, often with vague nodularity but no true follicular structure. Scattered individual epithelioid histiocytes are a helpful diagnostic feature (Figure 11) Cyclin D1 can be identified within nuclei using an immunostain (Figure 12). The cells bear surface IgM and there is a loose meshwork of follicular dendritic cells (CD21 stain), both characteristics of the normal follicular mantle.

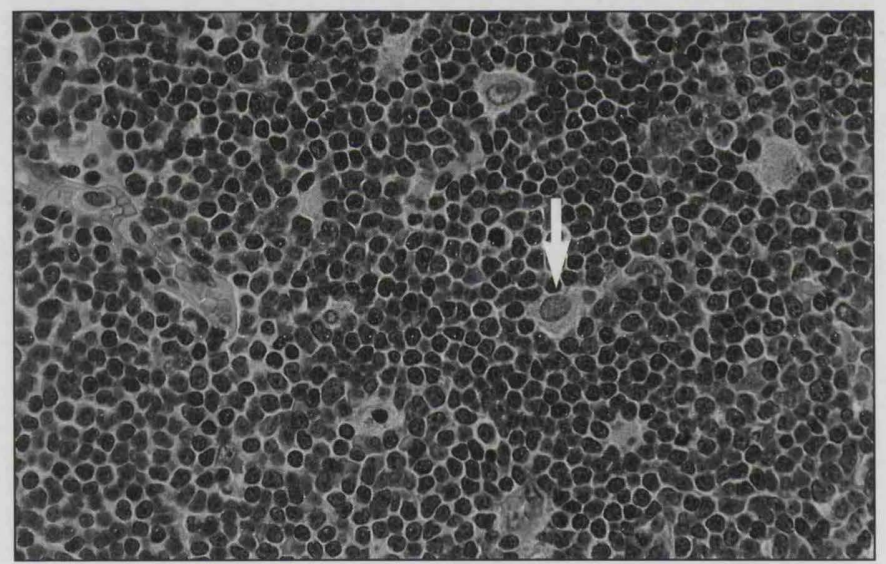

Figure 11 Mantle cell lymphoma. A monotonous infiltrate of small lymphocytes with scattered epithelioid histiocytes (arrow)

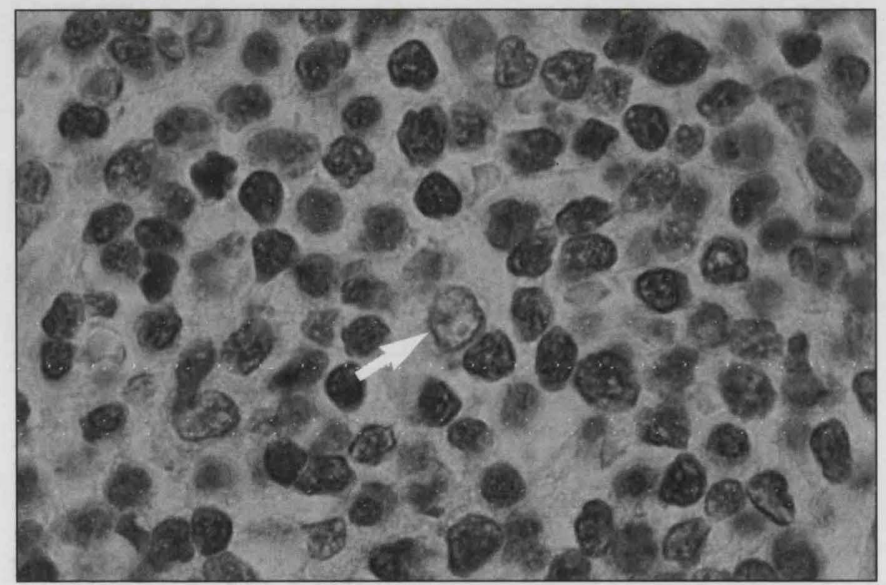

Figure 12 Mantle cell lymphoma. Small lymphocyte nuclei stain (dark) for cyclin D1. Note unstained histiocyte nucleus (arrow)

\section{Small lymphocytic lymphoma}

Lymphocytic lymphoma is the same disease as chronic ly mphocytic leukaemia and therefore affects the elderly. In the 
majority of cases the disease is leukaemic, but some cases present as lymphadenopathy and are then classified as small lymphocytic lymphoma. At diagnosis the disease is usually widespread in lymph nodes, bone marrow, spleen and peripheral blood.

The tumour probably represents a clonal expansion of the pool of recirculating small lymphocytes, which would account for its distribution. The tumour uncommonly affects extralymphoid sites. Small lymphocytic lymphoma is slowgrowing and incurable. Transformation into diffuse large Bcell lymphoma can occasionally occur. Histology is of a uniform infiltrate of small lymphocytes containing rather vague paler areas termed 'proliferation centres' where the cells are somewhat larger (Figure 13). The proliferation centres can be distinguished from true follicles by the absence of follicular dendritic cells (CD21 stain).

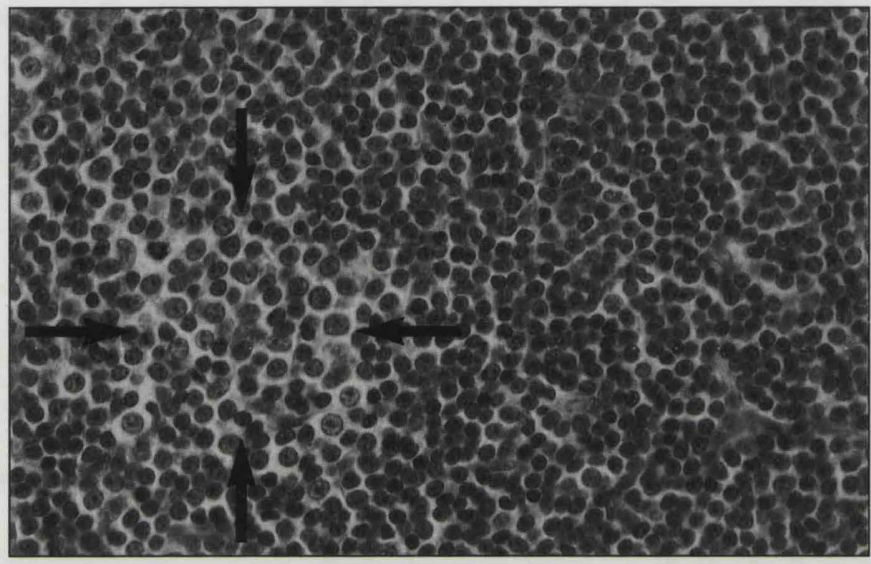

Figure 13 Proliferation centre in small lymphocytic lymphoma (arrows)

\section{Diffuse large B-cell lymphoma}

This is one of the common types of lymphoma and the name is more or less self-explanatory. There are no follicular structures and no nodularity (i.e. it is 'diffuse') and the lymphocytes are large. Nuclei are also large with an open chromatin structure, often with prominent nucleoli (Figure 14), unlike the small dense featureless nuclei of the lowgrade B-cell lymphomas.

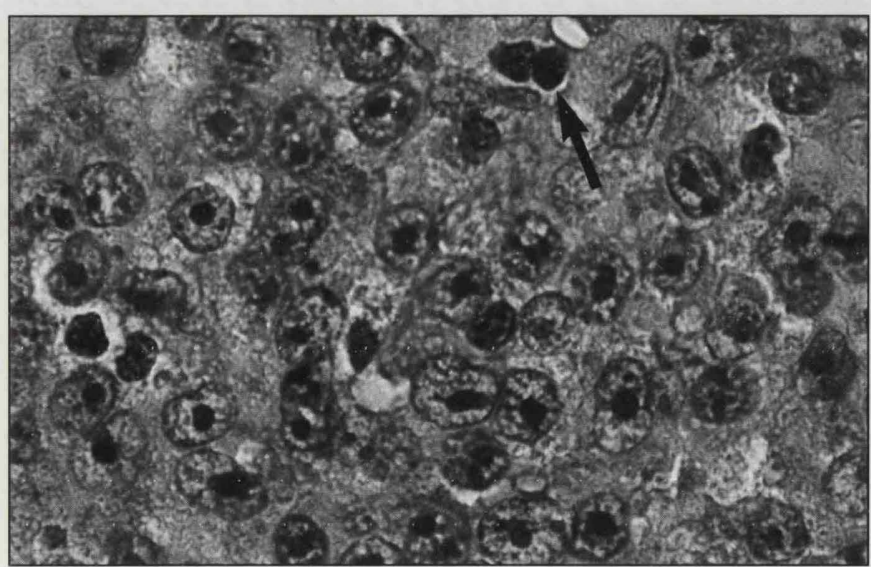

Figure 14 Diffuse large B-cell lymphoma (small lymphocytes arrowed for comparison)

Diffuse large B-cell lymphomas form rapidly-growing masses which tend to be more discrete and localised than low-grade B-cell lymphoma.

Most develop in lymph nodes, but $40 \%$ are extranodal, affecting a wide range of organs including the stomach, brain, and even soft tissue. A sizeable proportion of cases (at least
$30 \%$ ) arise as a result of high-grade transformation of a preexisting low-grade B-cell lymphoma.

Diffuse large B-cell lymphoma is an aggressive tumour, but is potentially curable by intensive chemotherapy with or without radiotherapy.

\section{Peripheral T-cell lymphoma}

This tumour primarily affects lymph nodes, but is a much less common cause of lymphadenopathy than the B-cell lymphomas. Peripheral T-cell lymphoma usually behaves aggressively, but a few can be cured by intensive therapy. The term 'peripheral' means mature.

Tumour cells usually comprise both large and small lymphocytes. A clue to the T-cell nature of the tumour is often provided by prominent vascularity and a mixture of reactive inflammatory cells such as eosinophils, plasma cells and histiocytes, all probably a response to lymphokines secreted by the tumour cells. Occasionally blood eosinophilia may occur.

\section{Anaplastic large cell lymphoma}

This is an uncommon recently-recognised type of lymphoma with the following diagnostic characteristics:

- large, usually pleomomorphic lymphocytes

- T-cell or null-cell phenotype

- tumour cells stain for CD30 (see information box).

Tumour cells frequently contain bizarre nuclei, often horseshoe-shaped or wreathlike with prominent nucleoli. Nuclei may be multiple and cells can resemble ReedSternberg cells. Unlike Hodgkin's disease, anaplastic large cell lymphoma forms diffuse sheets of large cells and tends to infiltrate lymph node sinuses; by contrast common types of Hodgkin's disease are nodular.

Anaplastic large cell lymphoma causes lymphadenopathy or can form tumour nodules in a wide range of extranodal sites. The tumour behaves aggressively, like diffuse large Bcell lymphoma, but is potentially curable by intensive therapy.

Many cases of anaplastic large cell lymphoma show a 2;5 chromosome translocation, causing production of an abnormal form of a protein p 80 (see Part II). This can now be detected in sections with a new immunostain ALK-1 ${ }^{(7)}$.

It is becoming clear that there are several subtypes ${ }^{(4,7)}$ of anaplastic large cell lymphoma (Figure 15).

\begin{tabular}{|l|c|c|c|}
\hline distribution & $2 ; 5$ translocation & age goup & prognosis \\
\hline systemic & + & $\begin{array}{c}\text { children } \\
\text { and } \\
\text { young } \\
\text { adults }\end{array}$ & quite good \\
\hline systemic & - & elderly & poor \\
\hline cutaneous only & - & any & good \\
\hline
\end{tabular}

Figure 15 Subtypes of anaplastic large cell lymphoma (* described in Part IV)

Anaplastic large cell lymphoma can occur as high-grade transformation of a low-grade lymphoma of either B-cell type ${ }^{(5)}$ or mycosis fungoides ${ }^{(8)}$. This 'secondary anaplastic large cell lymphoma' is believed to have a poor prognosis ${ }^{(8)}$. 
CD30 is one of many immunostains useful in lymphoma diagnosis. 'CD' means 'cluster of differentiation' and is used to define a series of different antigens in cells derived from bone marrow, which of course includes lymphocytes. At present almost 200 different antigens have been recognised and given a $C D$ number. Our immunostains include monoclonal antibodies which recognise specific $C D$ antigens, e.g. CD79a identifies $B-$ lymphocytes, CD3 identifies T-lymphocytes.

The fact that an antigen has been awarded a CD number does not mean that much is known about the structure and function of the antigen, only that it exists. Not much is known about $\operatorname{CD} 30$, but it is becoming increasingly useful in lymphoma diagnosis.

The CD30 story begins in 1982 when Stein produced a monoclonal antibody, named by him $\mathrm{Ki}-1$, which reacted with Reed-Sternberg cells, the origin of which he was investigating. $\mathrm{Ki}-1$ antibody was subsequently found also to stain activated benign lymphocytes and a type of large cell Iymphoma, which was termed anaplastic large cell Iymphoma ${ }^{(6)}$. The target for the $\mathrm{Ki}-1$ antibody was in due course designated as CD30. Ki-1 is the older term: CD30positive lymphomas and $\mathrm{Ki}-1$ lymphomas are synonymous.

At present CD30 is known to be an antigen expressed on activated $T$ and $B$ lymphocytes. It is believed to be responsible for the transduction of a signal for apoptosis $^{(2)}$.

Information box: $C D 30$

\section{HODGKIN'S DISEASE ${ }^{(1)}$}

Hodgkin's disease is a type of lymphoma characterised by large pleomorphic malignant cells mixed with and usually vastly outnumbered by reactive inflammatory cells. The classical Reed-Sternberg cell (Figure 16), with its mirrorimage double nuclei, and huge nucleoli, is merely one manifestation of the tumour cells.

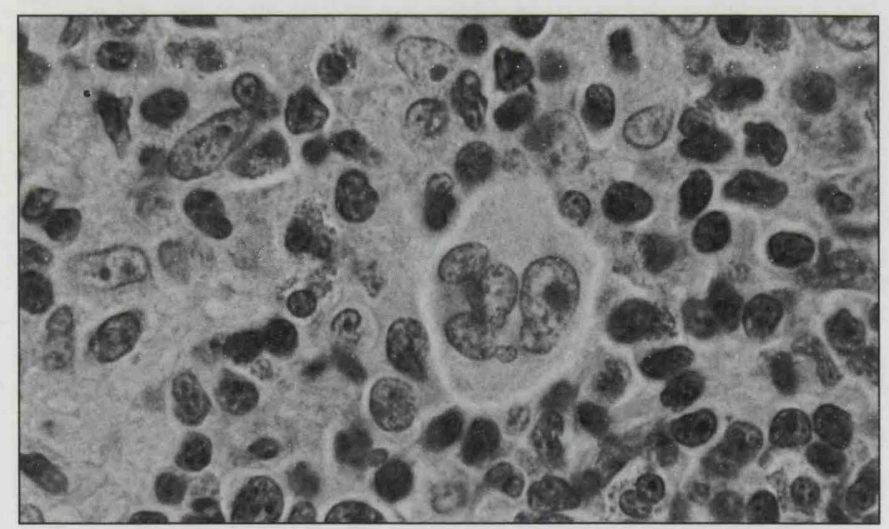

Figure 16 Reed-Sternberg cell in Hodgkin's disease. Note huge nucleoli

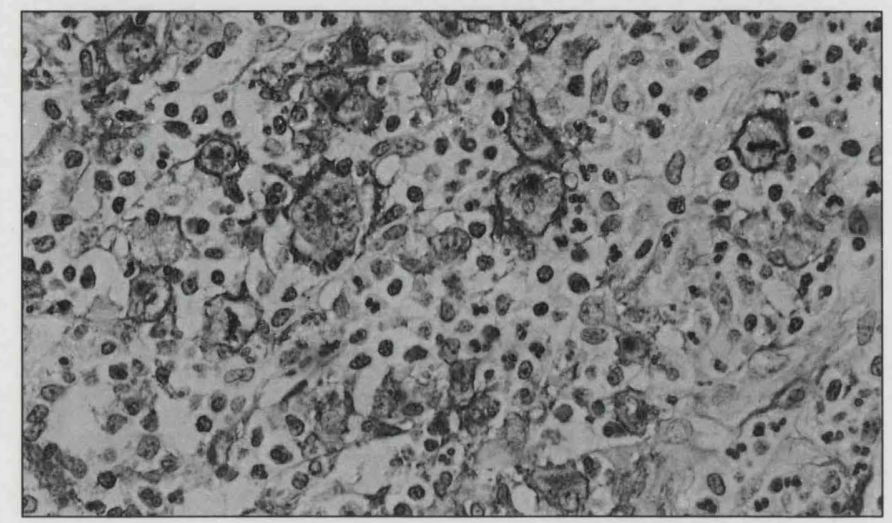

Figure 17 Reed-Sternberg cells stained for CD30
The malignant cells in most types of Hodgkin's disease have a null-cell phenotype and are CD30-positive (Figure 17), and there is therefore an obvious resemblance to anaplastic large lymphoma. The rare lymphocyte-predominance form of Hodgkin's disease is now known to have a B-cell phenotype (see below). In fact Hodgkin's disease is becoming increasingly difficult to define. In recent years the diagnostic boundary between Hodgkin's disease and non-Hodgkin's lymphoma has shifted somewhat in favour of the latter. Perhaps one day 'Hodgkin's disease' will disappear. Today it should be regarded as a special type of low-to-medium-grade lymphoma, which is often curable, almost always nodal, tends to affect young adults and is often associated with EB virus. This is reason enough to perpetuate Thomas Hodgkin's name.

Hodgkin's disease: lymphocyte-predominance

This is quite a rare type of Hodgkin's disease, and, since the malignant cells can now clearly be shown to have a B-cell phenotype (also negative for CD30), should perhaps be considered one of the B-cell lymphomas. The peak incidence is a decade later than other types of Hodgkin's disease. There is no association with $\mathrm{EB}$ virus.

The histology is of a nodular infiltrate of small benign Blymphocytes containing scanty scattered large malignant cells. T-lymphocytes often form rosettes around the malignant cells. Prognosis is good if the disease is localised, but late relapses are common, and there is a small risk of high-grade transformation into diffuse large B-cell lymphoma, a phenomenon not seen in other types of Hodgkin's disease.

\section{Hodgkin's disease: nodular sclerosis}

Nodular sclerosis is much the commonest type of Hodgkin's disease. The name is an accurate description of the histology. The lymphoma mass is divided into nodules by thick fibrous bands. The Reed-Sternberg cells are accompanied by a wide range of reactive inflammatory cells and show the typical null-cell phenotype and positive staining for CD30. Tumour cells contain EB virus in $40 \%$ of cases.

The tumour primarily affects lymph nodes, frequently in the neck and mediastinum, but often spreads to the liver, spleen and bone marrow. The disease is often curable by intensive therapy. Prognosis depends on bulk of disease and extent of spread (stage).

\section{Hodgkin's disease: mixed cellularity}

This is an uncommon type of Hodgkin's disease, in many ways comparable to nodular sclerosis, but lacking the fibrous bands.

\section{Hodgkin's disease: lymphocyte depletion}

In this type of Hodgkin's disease the tumour cells are the predominant feature, instead of the mixed inflammatory cells. Most cases are diagnosed nowadays as anaplastic large cell lymphoma.

\section{CONCLUSION}

This concludes our review of lymphoma classification and node-based lymphomas. In Part IV we shall consider extranodal lymphomas, their special features and common examples. 


\section{REFERENCES}

1 Chan JKC et al A proposal for classification of lymphoid neoplasms Histopathol 1994; 25:517-536

2 Roitt I Essential Immunology 9th ed Blackwell Science 1997

3 MacLennan I Germinal centres Ann Rev Immunol 1994; 12:117-139

4 Nakamura S et al Anaplastic large cell lymphoma: a distinct molecular pathologic entity Am J Surg Pathol 1997; $21: 1420-1432$

5 Alsabeh R, Medeiros LJ, Glackin C, Weiss LM Transformation of follicular lymphoma into CD30-large cell lymphoma with anaplastic cytologic features Am J Surg Pathol 1997; 21:528536
6 Stein H, Mason DY, Gerdes J et al The expression of the Hodgkin's disease associated antigen $\mathrm{Ki}-1$ in reactive and neoplastic lymphoid tissue: evidence that Reed-Sternberg cells and histologic malignancies are derived from activated lymphoid cells Blood 1985; 66:845-858

7 Shiota M, Mori S Anaplastic large cell lymphomas expressing the novel chimeric protein p80 NPM/ALK: a distinct clinicopathologic entity Leukaemia 1997; 11 (Suppl 3): $538-540$

8 Isaacson PG, Norton AJ Extranodal lymphomas Churchill Livingstone 1994 